YITP-SB-04-61

hep-th/0411171

\title{
Orbifolding the Twistor String
}

\author{
S. Giombi, M. Kulaxizi, R. Ricci, \\ D. Robles-Llana, D. Trancanelli and K. Zoubos ${ }^{1}$ \\ C. N. Yang Institute for Theoretical Physics, \\ State University of New York at Stony Brook \\ Stony Brook, NY 11794-3840, USA
}

\begin{abstract}
The D-instanton expansion of the topological B-model on the supermanifold $C P^{(3 \mid 4)}$ reproduces the perturbative expansion of $\mathcal{N}=4$ Super YangMills theory. In this paper we consider orbifolds in the fermionic directions of $C P^{(3 \mid 4)}$. This operation breaks the $S U(4)_{R} R$-symmetry group, reducing the amount of supersymmetry of the gauge theory. As specific examples we take $\mathcal{N}=1$ and $\mathcal{N}=2$ orbifolds and obtain the corresponding superconformal quiver theories. We discuss the D1 instanton expansion in this context and explicitly compute some amplitudes.
\end{abstract}

November 2004

\footnotetext{
${ }^{1}$ E-mails: sgiombi, kulaxizi, rricci, daniel, dtrancan, kzoubos@insti.physics.sunysb.edu
} 


\section{Contents}

1 Introduction $\quad 1$

2 Branes transverse to orbifold singularities $\quad 3$

2.1 Open string sector . . . . . . . . . . . . . . . . . . 3

2.2 Closed string sector . . . . . . . . . . . . . . . . . . . . . 4

3 The orbifold of the twistor string $\quad 5$

3.1 Review of the topological B-model on $C P^{(3 \mid 4)} \ldots \ldots$. . . . . . . . . 5

3.2 The orbifold . . . . . . . . . . . . . . . . . . . . . . . 6

3.3 D1 branes . . . . . . . . . . . . . . . . . . . . . . . 8

4 Explicit examples of orbifolds 12

4.1 An $\mathcal{N}=1$ orbifold . . . . . . . . . . . . . . . . . . . . . . . . . . . . . 12

4.1.1 MHV Amplitudes for the $\mathcal{N}=1$ orbifold . . . . . . . . . . . . . . . 14

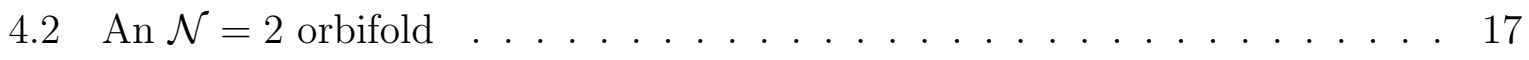

4.2.1 MHV Amplitudes for the $\mathcal{N}=2$ orbifold . . . . . . . . . . . . . . 17

5 Conclusion $\quad 18$

\section{Introduction}

The conjecture by Witten [1] that perturbative $\mathcal{N}=4$ super Yang-Mills can be realized as a D-instanton expansion of the topological B-model with $C P^{(3 \mid 4)}$ super-twistor space as target has generated a lot of interest recently. By now tree-level amplitudes are moderately well understood, either directly from the structure of the D-instanton moduli space [2], or from the twistor-inspired field theory procedure of $[3]^{2}$, which were shown to be related in [6]. More recently, some substantial progress has also been achieved in understanding one-loop amplitudes [7. Interesting alternative proposals to Witten's construction have been put forward in [8], starting from conventional open strings moving in $\left.C P^{(3 \mid 4)}, 9\right]$, where a mirror symmetric A-model version is considered, and [10], where ADHM twistors are considered. Conformal supergravity has been studied in this approach in [11] and [12], while the possibility of extending the twistor formalism to ordinary gravity has been investigated in 13. Other recent related work can be found in [14.

Surprising and elegant as Witten's proposal undoubtedly is, it has two obvious shortcomings. The first one is that so far it applies only to maximally supersymmetric gauge

\footnotetext{
${ }^{2}$ See [4] for subsequent developments and [5] for a review.
} 
theories ${ }^{3}$; the second is that superconformal invariance is automatically built-in by virtue of the twistor formalism. These features seems to make the original construction unfit for describing more realistic gauge theories. The problem of reducing the model to an $\mathcal{N}=1$ superconformal theory has been recently considered in [15], where a Leigh-Strassler deformation of $\mathcal{N}=4 \mathrm{SYM}$ was implemented via open/closed couplings.

In this paper we consider a different extension of Witten's correspondence to a class of $\mathcal{N}=2$ and $\mathcal{N}=1$ supersymmetric gauge theories. As we do not know how to relax the requirement of superconformal invariance, natural candidates are the superconformal quiver theories analyzed in [16] and [17]: In this paper we will recover them from twistor strings.

The procedure followed in [16] and [17] was to start with a parent $\mathcal{N}=4$ super Yang-Mills theory and retrieve the superconformal daughter theories with reduced supersymmetry by orbifolding the $S U(4)_{R}$ symmetry rotating the supercharges ${ }^{4}$. These are quiver theories with bifundamental matter fields. In the present case of twistor strings, the $S U(4)_{R}$ symmetry is part of the isometry group of $C P^{(3 \mid 4)}$. Thus, before Penrose transforming, this operation has a natural interpretation as a fermionic orbifold of the twistor string's target space.

Although it is not clear a priori what the meaning of a fermionic orbifold is, one is immediately tempted to establish a connection with the standard lore about D-branes tranverse to bosonic orbifold singularities [18, and their realization via geometric engineering [19]. In the case of $\mathcal{N}=2$ superconformal theories engineered from type IIB superstrings ${ }^{5}$, the moduli space of superconformal couplings is known to admit a duality group whose action is inherited from the S-duality of $I I B$ superstrings ${ }^{6}$. It would be interesting to identify these moduli spaces in the twistor string theory.

This paper is organized as follows: In Section 2, we briefly recall basic facts about D-branes on (bosonic) orbifolds. In Section 3, after reviewing the twistor construction of [1, we perform the orbifold on the fermionic directions of the super-twistor space, paying particular attention to the role of the $D 1$ branes. Consistency will require the introduction of $|\Gamma|$ D1 branes, where $|\Gamma|$ is the order of the orbifold group. In Section 4, we investigate two examples of $\mathcal{N}=1$ and $\mathcal{N}=2$ orbifolds, and explicitly compute some amplitudes. In Section 5, we give the conclusion and some final remarks.

\footnotetext{
${ }^{3}$ However, examples of twistor-inspired computations of amplitudes in theories with less supersymmetry have appeared in the literature.

${ }^{4}$ Strictly speaking, this is not really an orbifold in the conventional sense of the word, as one is not gauging the discrete symmetry in spacetime.

${ }^{5}$ These are necessarily of affine $A D E$ type.

${ }^{6}$ Alternatively, it can be seen as the affine Weyl group acting on the primitive roots of affine $A D E$ group [20] 21] [17.
} 


\section{Branes transverse to orbifold singularities}

Placing a set of $D$ branes transverse to a $C^{n} / \Gamma$ orbifold (where $n=2,3$, and $\Gamma$ is a discrete subgroup of $S U(n))$ gives rise to four dimensional gauge theories with $\mathcal{N}=2$ or $\mathcal{N}=1$ supersymmetry living in the brane worldvolume. To obtain their massless spectrum one has to consider the appropriate orbifold action on fields in both the open and closed string sectors. The open string sector contributes the field content of the gauge theories, which can be encoded in quiver diagrams. The closed string sector in turn contributes the necessary moduli which deform the transverse orbifold singularity to a smooth space. We briefly review this construction below, focusing in the case of abelian $\Gamma$ for simplicity.

\subsection{Open string sector}

In the open string sector, $\Gamma$ acts on the orbifolded transverse coordinates and on the ChanPaton factors of open strings in the worldvolume directions. To get conformal theories one needs $\Gamma$ to act on the Chan-Paton factors in (arbitrary number of copies of) the regular representation $\mathcal{R}$ of $\Gamma$. Choosing $N$ copies of $\mathcal{R}$ amounts to considering $N|\Gamma| D$ 3-branes before the projection, where $|\Gamma|$ is the order of $\Gamma$. At this stage one then has $U(N|\Gamma|)$ gauge symmetry in the worldvolume.

In the worldvolume directions $\Gamma$ acts on the open string Chan-Paton factors matrices $\lambda$ only. This action is specified by a matrix $\gamma_{\Gamma} \in \mathcal{R}$, and the invariant states satisfy

$$
\gamma_{\Gamma} \lambda \gamma_{\Gamma}^{-1}=\lambda
$$

Now using elementary group theory the regular representation can be decomposed in irreducible representations as

$$
\mathcal{R}=\oplus_{\mathbf{i}} n_{\mathbf{i}} \mathcal{R}_{\mathbf{i}}
$$

where $n_{\mathbf{i}}=\operatorname{dim}\left(\mathcal{R}_{\mathbf{i}}\right)=1$ for abelian $\Gamma$. Acting on $\lambda$ as in (2.1) this decomposition projects out the fields whose Chan-Paton indices are not connected by one of the irreducible $\mathcal{R}_{\mathrm{i}}$ 's. Taking $N$ copies of $\mathcal{R}$ the gauge group will therefore be broken ${ }^{7}$ to $F=\prod_{\mathbf{i} \in \text { irreps }} U(N)$. Each of these unitary groups with its gauge multiplet has an associated node in the quiver diagram.

In the orbifolded directions $\Gamma$ acts on the Chan-Paton matrices through an element of the regular representation $\gamma_{\Gamma}$, and on space indices through the defining $n$ dimensional representation $G_{\Gamma}^{n \times n}$, in such a way that

$$
G_{\Gamma}^{n \times n}(\Psi(\mathbf{i}))=\Psi\left(\gamma_{\Gamma}(\mathbf{i})\right)
$$

${ }^{7}$ Actually there is a $U(1)$ subgroup acting trivially on the fields. It can be seen as the motion of the center of mass coordinate of the D-branes. Therefore the effective gauge group is $G=F / U(1)$. 
$\Psi(\mathbf{i})$ being a label for the $\mathbf{i}$-th $D 3$-brane in the orbifolded transverse space. This means that the action of the space group is correlated with the action on the Chan-Paton factors. The fields surviving the projection (2.3) can be obtained from the decomposition

$$
\begin{aligned}
\operatorname{Hom}\left(\mathcal{R}, G_{\Gamma}^{n \times n} \otimes \mathcal{R}\right) & =\bigoplus_{\mathbf{i}, \mathbf{j}}\left[\operatorname{Hom}\left(\mathcal{R}_{\mathbf{i}}, G_{\Gamma}^{n \times n} \otimes \mathcal{R}_{\mathbf{j}}\right) \otimes \operatorname{Hom}\left(C^{N}, C^{N}\right)\right] \\
& =\bigoplus_{\mathbf{i}, \mathbf{j}} a_{\mathbf{i} \mathbf{j}} \operatorname{Hom}\left(C^{N}, C^{N}\right)
\end{aligned}
$$

where again $\mathbf{i}$ runs over irreducible representations, and $a_{\mathbf{i j}}$ are the Clebsch-Gordan coefficients in the decomposition of the tensor product. Physically these are $a_{\mathbf{i j}}$ chiral multiplets transforming in bifundamental representations as

$$
\oplus a_{\mathbf{i j}}(\mathbf{N}, \overline{\mathbf{N}})
$$

The quiver diagram has $a_{\mathbf{i j}}$ oriented links between nodes $\mathbf{i}$ and $\mathbf{j}$. For $\mathcal{N}=2$ quivers $a_{\mathbf{i j}}=a_{\mathbf{j i}}$, which makes the links non oriented. Each of them represents an $\mathcal{N}=2$ hypermultiplet.

Finally, for $C^{2} / \Gamma$ orbifolds one has two non-orbifolded transverse directions. $\Gamma$ acts on these fields as in (2.1). They provide the adjoint chiral superfields which together with the gauge multiplets complete the $\mathcal{N}=2$ vector multiplet.

\subsection{Closed string sector}

In the closed string sector there are no Chan-Paton factors, and one can follow the ordinary orbifold techniques to find the spectrum. There are, in addition to the usual untwisted sector, $|\Gamma|-1$ twisted sectors which play a crucial role in resolving the singularity. The untwisted sector is just the Kaluza-Klein reduction of the ten dimensional supergravity multiplet on $C^{2} / \Gamma$ (for $\mathcal{N}=2$ ) or on $C^{3} / \Gamma$ (for $\mathcal{N}=1$ ), together with the usual matter multiplets. In the large volume limit the moduli fields from the $|\Gamma|-1$ twisted sectors can be seen to arise by wrapping the various form fields on the exceptional cycles of the blown-up singularity. For $\mathcal{N}=2$ the blow-up is hyper-Kähler, whereas for $\mathcal{N}=1$ it is only Kähler. In the first case there are moduli $b_{\mathbf{i}}=\int_{S_{\mathbf{i}}^{2}} B$ and $\vec{\zeta}_{\mathbf{i}}=\int_{S_{\mathbf{i}}^{2}} \vec{\omega}$, where for $I I A$ $B$ is the $N S N S$ B-field ${ }^{8}$, and $\vec{\omega}$ is the triplet of Kähler forms on the blow up. In the Kähler case $b_{\mathbf{i}}=\int_{S_{\mathbf{i}}^{2}} B$ and $\zeta_{\mathbf{i}}=\int_{S_{\mathbf{i}}^{2}} \omega$, where $\omega$ is the Kähler form. In the first case the combination $b_{\mathbf{i}}+i \vec{\zeta}_{\mathbf{i}}$ encodes (in the large volume limit) the deformations of Kähler and complex structure of the resolution. Because of $\mathcal{N}=2$ supersymmetry the resolution is

\footnotetext{
${ }^{8}$ For $I I B$ one can in addition have the two-form from the $R R$ sector.
} 
hyper-Kähler, and these two are related by the $S O(3)$ symmetry that rotates $\vec{\omega}$. In the second case $b_{\mathbf{i}}+i \zeta_{\mathbf{i}}$ parameterize the deformations of the complexified Kähler structure ${ }^{9}$.

Of course one has in addition to these moduli scalars various $p$-form fields from the twisted sectors. The twisted fields couple to open fields in the brane low effective action via Chern-Simons couplings. In the presence of the orbifold, closed fields from the $k$-th twisted sector $C_{k}$ couple naturally to the $U(1)$ part of the field strength of the $D$-brane whose Chan-Paton factor is twisted by $\gamma_{\Gamma}$. Their supersymmetric completion involves terms which couple as Fayet-Iliopoulos parameters in the effective gauge theory on the brane world-volume. When these are non-zero the gauge symmetry is completely broken, and the Higgs branch of the world-volume theory is the resolved transverse space.

Taking into account all the twisted moduli one can write the full stringy quantum volume of the exceptional cycles of the geometry as $V_{\mathbf{i}}=\left(b_{\mathbf{i}}^{2}+\left|\vec{\zeta}_{\mathbf{i}}\right|^{2}\right)^{1 / 2}$ in the $\mathcal{N}=2$ case and $V_{\mathbf{i}}=\left(b_{\mathbf{i}}^{2}+\zeta_{\mathbf{i}}^{2}\right)^{1 / 2}$ in the $\mathcal{N}=1$ case. At the orbifold point $\zeta_{\mathbf{i}}=0$, and one can write the coupling constant of the $\mathbf{i}$-th gauge group as $1 /\left(g_{Y M \mathbf{i}}\right)^{2}=V_{\mathbf{i}} / g_{s}$, where $g_{s}$ is the string coupling constant. In type $I I B$ one has also $c_{\mathbf{i}}=\int_{S_{\mathbf{i}}^{2}} B_{R}$, which plays the role of a theta angle in the gauge theory. One can then write the complexified couplings as $\tau_{\mathbf{i}}=\theta_{\mathbf{i}}+i /\left(g_{Y M \mathbf{i}}\right)^{2}=c_{\mathbf{i}}+b_{\mathbf{i}} \tau$, with $\tau=g_{s}^{-1}$. The S-duality of type $I I B$ superstrings, which acts on $B_{N S}$ and $B_{R}$ manifests itself as a duality in the moduli space of couplings.

\section{The orbifold of the twistor string}

\subsection{Review of the topological B-model on $C P^{(3 \mid 4)}$}

We start by reviewing the model proposed by Witten [1] to describe perturbative $\mathcal{N}=4$ SYM: This is a topological B-model on the supermanifold $C P^{(3 \mid 4)}$. The local bosonic and fermionic coordinates on this space are $\left(Z^{I}, \psi^{A}\right)$, with $I=1, \ldots, 4$ and $A=1, \ldots, 4$. They are subject to the identification $\left(Z^{I}, \psi^{A}\right) \sim\left(t Z^{I}, t \psi^{A}\right)$, with $t \in C^{*}$. The $Z^{I}$ cannot be all zero. Equivalently, this supermanifold can be defined as the sublocus of $C^{(4 \mid 4)}$

$$
\left(\sum_{I}\left|Z^{I}\right|^{2}+\sum_{A}\left|\psi^{A}\right|^{2}=r\right) / U(1)
$$

where $U(1)$ is a phase transformation acting as $\left(Z^{I}, \psi^{A}\right) \rightarrow e^{i \alpha}\left(Z^{I}, \psi^{A}\right)$. This space is a super Calabi-Yau with holomorphic volume form

$$
\Omega=\frac{1}{(4 !)^{2}} \epsilon_{I J K L} \epsilon_{A B C D} Z^{I} d Z^{J} d Z^{K} d Z^{L} d \psi^{A} d \psi^{B} d \psi^{C} d \psi^{D} .
$$

\footnotetext{
${ }^{9}$ In this case, in addition to $B$ there are further moduli from the $R R$ sector: a hypermultiplet for type $I I B$ or a vector multiplet for type $I I A$.
} 
In this model the self-dual part of $\mathcal{N}=4 \mathrm{SYM}$ with gauge group $U(N)$ is reproduced by the world volume action of a stack of $N$ D 5 branes. These are almost space-filling branes placed at $\bar{\psi}_{A}=0$. The world volume action is a holomorphic Chern-Simons

$$
\mathcal{S}=\int_{D 5} \Omega \wedge \operatorname{Tr}\left(\mathcal{A} \bar{\partial} \mathcal{A}+\frac{2}{3} \mathcal{A} \wedge \mathcal{A} \wedge \mathcal{A}\right)
$$

where $\mathcal{A}=\mathcal{A}_{\bar{I}} d \bar{Z}^{\bar{I}}$ is a $(0,1)$ form with values in the adjoint representation of $U(N)$. The superfield expansion reads

$$
\begin{aligned}
\mathcal{A}(z, \bar{z}, \psi) & =A(z, \bar{z})+\psi^{A} \chi_{A}(z, \bar{z})+\frac{1}{2} \psi^{A} \psi^{B} \phi_{A B}(z, \bar{z}) \\
& +\frac{1}{3 !} \epsilon_{A B C D} \psi^{A} \psi^{B} \psi^{C} \tilde{\chi}^{D}(z, \bar{z})+\frac{1}{4 !} \epsilon_{A B C D} \psi^{A} \psi^{B} \psi^{C} \psi^{D} G(z, \bar{z}) .
\end{aligned}
$$

The components of $\mathcal{A}$ are charged under the symmetry

$$
S: \psi^{A} \rightarrow e^{i \beta} \psi^{A}
$$

as $S(A, \chi, \phi, \tilde{\chi}, G)=(0,-1,-2,-3,-4)$. One can integrate out the fermionic coordinates to get

$$
\begin{aligned}
\mathcal{S} & =\int_{C P^{3}} \Omega^{\prime} \wedge \operatorname{Tr}\left[G \wedge(\bar{\partial} A+A \wedge A)+\tilde{\chi}^{A} \wedge \bar{D} \chi_{A}\right. \\
& \left.+\frac{1}{4} \epsilon^{A B C D} \phi_{A B} \wedge \bar{D} \phi_{C D}+\frac{1}{2} \epsilon^{A B C D} \chi_{A} \wedge \chi_{B} \wedge \phi_{C D}\right]
\end{aligned}
$$

where $\Omega^{\prime}$ is the bosonic reduction of $\Omega$. After performing a Penrose transform [22], (3.4) yields the field content of the $\mathcal{N}=4$ vector multiplet, whereas (3.6) reproduces the self-dual truncation of the $\mathcal{N}=4$ SYM action [23].

The non self-dual completion of the theory is obtained by introducing D1 instanton corrections. These are holomorphic maps from $C P^{1}$ to $C P^{3 \mid 4}$ and the instanton number corresponds to the degree of the map. We will discuss later in more detail the role played by these D1 branes.

\subsection{The orbifold}

The procedure reviewed in Section 2 can be applied to the twistor string of [1] in order to reduce the $\mathcal{N}=4$ supersymmetry. The homogeneous coordinates of $C P^{3 \mid 4}$ provide a linear realization of $P S U(4 \mid 4)$, which is the $\mathcal{N}=4$ superconformal group. It is therefore natural to use twistors to study conformal theories. To reduce supersymmetry, we can orbifold the fermionic directions of the super-twistor space. Physically, this amounts to orbifolding the $S U(4)_{R} R$-symmetry, which is the Fermi-Fermi subgroup of $P S U(4 \mid 4)$. As reviewed above, in the twistor theory of [1] a set of D5 branes is placed at $\bar{\psi}_{A}=0$. In 


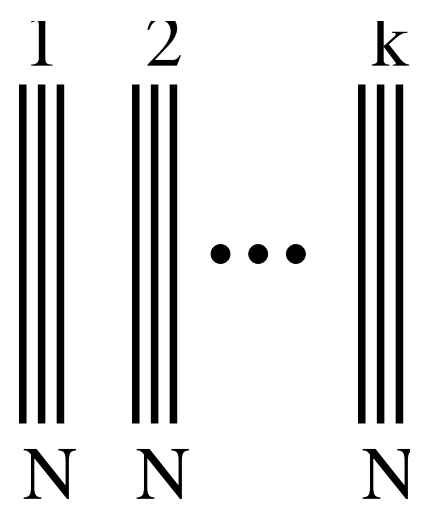

Figure 1: Regrouping of the D5 branes under the orbifold action. Note that the $k$ stacks of branes are actually coincident at the point $\bar{\psi}_{A}=0$.

analogy with the conventional case, one possible interpretation is to view the orbifold as acting in the $\bar{\psi}_{A}$ directions, which are transverse to the D5 branes. This induces an action on the $\psi^{A}$ which will be the one considered in the following.

Explicitly, we choose an action of the orbifold $\Gamma \in Z_{k}$ under which the fermionic coordinates transform as

$$
\psi^{A} \rightarrow e^{2 \pi i a_{A} / k} \psi^{A}
$$

with the condition on the charges $\sum_{A} a_{A}=0(\bmod k)$, so that $\Gamma \in S U(4)_{R}$. The holomorphic volume form (3.2) is invariant under (3.7): This implies that the superorbifold is still Calabi-Yau. This is crucial for the consistency of the B-model [24].

We consider a stack of $k N$ D5 branes in the covering space. The orbifold action regroups the branes in $k$ stacks of $N$ branes each, as shown in Figure 1. It is thus convenient to decompose the $U(k N)$ adjoint index into $A_{b}^{a}=A_{J \mathbf{j}}^{I \mathbf{i}}$, where $I, J=1, \ldots, N$ label the brane within a stack and $\mathbf{i}, \mathbf{j}=1, \ldots, k$ label the stacks.

An explicit representation of $\Gamma$ is

$$
\mathcal{R}=\left(\begin{array}{cccc}
r_{1} & 0 & \cdots & 0 \\
0 & r_{2} & \cdots & 0 \\
\vdots & \vdots & \ddots & \vdots \\
0 & 0 & \cdots & r_{k}
\end{array}\right)_{k N \times k N}
$$

where $r_{\mathbf{i}}=e^{2 \pi i \mathbf{i} / k}$ is a $N \times N$ matrix acting on the $\mathbf{i}$-th node of the associated quiver. The orbifold projection is enforced by requiring invariance of the components of the superfield $\mathcal{A}$ under the action of $\Gamma$. $R$-symmetry invariance of the superfield implies that (3.7) induces a conjugate transformation on the fermionic indices of the components. Combined 
with the action on the Chan-Paton factors given by (3.8), this gives the following orbifold action on a generic component (with $n=0, \ldots, 4$ fermionic indices) ${ }^{10}$

$$
\left(\Phi_{A_{1}, \ldots, A_{n}}\right)_{J \mathbf{j}}^{I \mathbf{i}} \rightarrow e^{2 \pi i\left(\mathbf{i}-\mathbf{j}-a_{A_{1}}-\ldots-a_{A_{n}}\right) / k}\left(\Phi_{A_{1}, \ldots, A_{n}}\right)_{J \mathbf{j}}^{I \mathbf{i}}
$$

For example, the lowest component of (3.4), which physically represents the positive helicity gluon, transforms as

$$
A_{J \mathbf{j}}^{I \mathbf{i}} \rightarrow e^{2 \pi i(\mathbf{i}-\mathbf{j}) / k} A_{J \mathbf{j}}^{I \mathbf{i}}
$$

Invariance requires $\mathbf{i}=\mathbf{j}$, so that the gauge group is broken to

$$
U(k N) \rightarrow[U(N)]^{k}
$$

Similarly, the positive helicity gluino $\chi_{A}$ transforms as

$$
\left(\chi_{A}\right)_{J \mathbf{j}}^{I \mathbf{i}} \rightarrow e^{2 \pi i\left(\mathbf{i}-\mathbf{j}-a_{A}\right) / k}\left(\chi_{A}\right)_{J \mathbf{j}}^{I \mathbf{i}}
$$

so that in this case one needs to enforce $\mathbf{i}=\mathbf{j}+a_{A}$. Depending on the value of the charge $a_{A}$, the field $\chi_{A}$ becomes either a gaugino or a bi-fundamental quark. One proceeds analogously with the other components of $\mathcal{A}$. One can picture the field content in a quiver diagram with $k$ nodes corresponding to the $k$ gauge groups and bi-fundamental matter as lines connecting pairs of nodes.

The choice of the discrete group one quotients by determines the amount of supersymmetry preserved by the orbifold. For generic $\Gamma$ the supersymmetry is completely broken, while for $\Gamma \in Z_{k} \subset S U(2)_{R}$ and $\Gamma \in Z_{k} \subset S U(3)_{R}$ one has respectively $\mathcal{N}=2$ and $\mathcal{N}=1$ [16].

So far we have only focused on the D5 brane sector. In the next Section we will tackle the problem of orbifolding the D1 instantons.

\subsection{D1 branes}

As already remarked, the holomorphic Chern-Simons action on $C P^{3 \mid 4}$ only reproduces the self-dual truncation of $\mathcal{N}=4$ SYM. A non-perturbative correction to the B-model is needed in order to recover the non self-dual part of the gauge theory. These new nonperturbative degrees of freedom are D1 branes wrapped on holomorphic cycles inside the supermanifold. These branes are D-instantons whose instanton number is given by the degree $d$ of the map. The simplest case ${ }^{11}$ is genus $g=0$ and $d=1$. This is the only

\footnotetext{
${ }^{10}$ In this notation, for instance, $\Phi_{A_{1} A_{2} A_{3} A_{4}}=\frac{1}{4 !} \epsilon_{A_{1} A_{2} A_{3} A_{4}} G$, where $G$ is the highest component of $\mathcal{A}$.

${ }^{11}$ We will only consider tree-level scattering amplitudes. Amplitudes with $l$ loops receive contributions also from curves of genus $g \leq l$.
} 
example we discuss in this paper. The explicit map is

$$
\begin{aligned}
& \mu_{\dot{a}}+x_{a \dot{a}} \lambda^{a}=0 \\
& \psi^{A}+\theta_{a}^{A} \lambda^{a}=0 .
\end{aligned}
$$

Here the coordinates of $C P^{3}$ are decomposed as $Z^{I}=\left(\lambda^{a}, \mu_{\dot{a}}\right)$ and $x_{a \dot{a}}, \theta_{a}^{A}$ are the moduli of the embedding. In [1] the D1 instanton is initially placed at $\psi^{A}=0$ and the dependence on the fermionic coordinates is then restored through an integration over the moduli space.

Tree-level gauge theory scattering amplitudes are computed by considering the effective action for D1-D5 strings

$$
I_{D 1-D 5}=\int_{D 1} d z \beta \bar{D} \alpha
$$

where $\alpha$ and $\beta$ are fermions which carry respectively fundamental and anti-fundamental gauge group indices. They correspond to strings stretching from the D1 to the D5 brane and viceversa. The covariant derivative is $\bar{D}=\bar{\partial}+\mathcal{A}$. The action (3.14) contains the interaction term

$$
\Delta I_{D 1-D 5}=\int_{D 1} \operatorname{Tr} J \mathcal{A}=\int_{D 1} J_{b}^{a} \mathcal{A}_{a}^{b}
$$

where $J_{b}^{a}=\alpha^{a} \beta_{b}$. Scattering amplitudes are obtained by taking correlation functions of the currents $J$ 's in the background of the superfield $\mathcal{A}$ and integrating them over the moduli space of a D1 instanton of appropriate degree. This degree is determined by the sum over the $S$ symmetry (3.5) charges of the $n$ external states

$$
d=-\frac{1}{4} \sum_{i=1}^{n} S_{i}-1
$$

In the particular case of external gluons, this corresponds to $d=q-1$, where $q$ is the number of negative helicity gluons. Explicitly, when $d=1$ one has for the $n$-point scattering amplitude

$$
A_{n}=\int d^{8} \theta w_{1} \ldots w_{n}\left\langle J_{1}^{a_{1}} \ldots J_{n}^{a_{n}}\right\rangle
$$

where the $w_{i}$ are the wave functions of the external states and are given essentially by the coefficient of that state in the superfield expansion. We stress that the measure in (3.17) is going to be invariant under the orbifold action.

We now discuss the issue of how the coupling constant may arise in the theory. From a four dimensional field theoretical point of view, the completion of the self-dual Yang-Mills action $I_{S D}=\int d^{4} x \operatorname{Tr}(G F)$ is given by

$$
I_{Y M}=\int d^{4} x \operatorname{Tr}\left(G F-\frac{g_{Y M}^{2}}{2} G^{2}\right) .
$$


Therefore, in the topological B-model one expects the coupling constant to originate from the D1 instanton expansion. This is rather surprising since now the YM perturbative coupling seems to come from non-perturbative sectors of the theory. One natural way to introduce a free parameter in the amplitude (3.17) is to weigh it by a factor $\left(e^{-I_{D 1}}\right)^{d}$, where $I_{D 1}$ is the action for a D1 instanton of degree $d=1$. This has been already remarked in [1. Another way to achieve this is to consider the coupling of the D1 to the closed sector of the B-model. This was first realized in [11] where a new field $b$, a $(1,1)$ form in twistor space, is introduced. It has a minimal coupling to the D1 worldvolume

$$
I_{b}=\int_{D 1} b_{I \bar{J}} d Z^{I} \wedge d \bar{Z}^{\bar{J}}
$$

This field is not present in the perturbative analysis of the B-model. The necessity of it was also recently rediscussed in [25] as a non-perturbative correction to Kodaira-Spencer theory [26]. For a D1 sitting at $(x, \theta)$ in the moduli space the coupling (3.19) directly defines the conformal supergravity superfield $\mathcal{W}(x, \theta)=\int_{D 1_{(x, \theta)}} b$. The lowest component can be interpreted as a dilaton $C$. As a consequence of the coupling (3.19), in a vacuum with expectation value $\langle C\rangle=c$, an amplitude will be weighted by a factor $\left(e^{-c}\right)^{d}$. This is reminiscent of ordinary string theory where the coupling constant comes from the dilaton expectation value.

In summary, we assume the contribution of the D1 instanton to an amplitude to be equal to $\left(g^{2}\right)^{d}$, where $g^{2}$ might come from $e^{-I_{D 1}}$ or $e^{-c}$.

To get the standard normalization of the scattering amplitudes, one also needs to rescale each component of the superfield $\mathcal{A}$ by a factor $g^{1+\frac{1}{2} S}$, where $S$ is the charge under the symmetry (3.5): For instance, $A$ goes to $g A, \chi$ to $\sqrt{g} \chi$, and so on. In the end, the overall coupling constant in a tree-level $n$-point amplitude is

$$
\left(\prod_{i=1}^{n} g^{1+\frac{1}{2} S_{i}}\right)\left(g^{2}\right)^{-\frac{1}{4} \sum_{i} S_{i}-1}=g^{n-2}
$$

We now proceed to the analysis of the orbifold action on the D1 instanton sector. For this action to be faithful on the Chan-Paton factors of the D1's, we need to start with $k$ D1 branes. To begin with, we locate them at $\psi^{A}=0$. As in [1], the fermionic dependence will be restored in the end through integration over the moduli space. Considering $k$ D1 branes, the effective action (3.14) gets changed into

$$
I_{D 1-D 5}=\int_{D 1} d z\left(\beta_{I \mathbf{i}}^{r} \bar{\partial} \alpha_{r}^{I \mathbf{i}}+\beta_{I \mathbf{i}}^{r} \mathcal{B}_{r}^{s} \alpha_{s}^{I \mathbf{i}}+\beta_{I \mathbf{i}}^{r} \mathcal{A}_{J \mathbf{j}}^{I \mathbf{i}} \alpha_{r}^{J \mathbf{j}}\right)
$$

where $r=1, \ldots, k$ is a $U(k)$ index which labels the D1's. For instance, $\alpha_{r}^{I \mathbf{i}}$ is a string stretching from the $r$-th D1 brane to the $I$-th D5 brane inside the $\mathbf{i}$-th stack. In (3.21) $\mathcal{B}_{r}{ }^{s}$ 

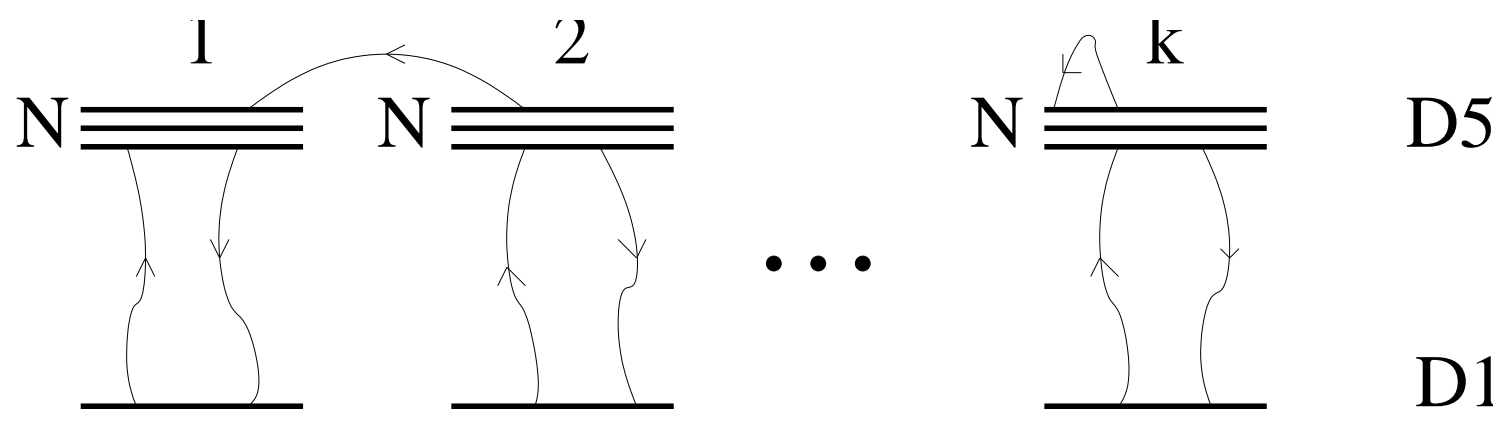

Figure 2: The stacks of D5 and D1 branes. An interaction between the first and the second stack is depicted.

is the $U(k)$ gauge field on the world-volume of the D1 branes. The action of the orbifold breaks $U(k) \rightarrow[U(1)]^{k}$. The D1-D5 strings $\alpha$ and $\beta$ transform as

$$
\begin{aligned}
& \alpha_{r}^{I \mathbf{i}} \rightarrow e^{2 \pi i(\mathbf{i}-r) / k} \alpha_{r}^{I \mathbf{i}} \\
& \beta_{I \mathbf{i}}^{r} \rightarrow e^{2 \pi i(r-\mathbf{i}) / k} \beta_{I \mathbf{i}}^{r} .
\end{aligned}
$$

Invariance under the orbifold action requires $\mathbf{i}=r$. This implies that the D1-D5 strings only stretch between the $\mathbf{i}$-th D1 brane and the D5 branes in the $\mathbf{i}$-th stack. This is shown in Figure 2 and, in quiver language, for the specific example of $k=3$, in Figure 3 .

The $U(1)$ fields living on the D1 branes and the bi-fundamental matter connecting them will not be considered in the following, although they are depicted in Figure 3 . The $U(1)$ bundles over curves of genus $g<2$ do not have moduli as remarked in [1] and do not play a role in the computation of amplitudes. Further, it seems natural to neglect the bi-fundamental fields since in general, when the branes move away from $\psi^{A}=0$, they should correspond to massive states.

The stack of $k$ D1 branes can move away from the orbifold fixed point as one full regular brane. In the covering space, the $k$ D1 branes are located in points related by the $\Gamma$ action in the orbifold directions, whereas they coincide in the others. In particular, they coincide along the bosonic subspace and therefore the bosonic world-volume is the same for all of them. Since the branes cannot move independently we have only one set of moduli $(x, \theta)$ for the whole system. However this is not the complete story. We do not fully explore the richness of the orbifold construction: If the branes were coincident at the fixed point of the fermionic coordinates, then there would be no constraint on their motion along the remaining directions and one would have an extended moduli space $\left\{\left(x_{r}, \theta_{r}\right)\right\}$ with $k$ sets of parameters. We will not study this explicitly but we will limit ourselves to some comments. Having $k$ independent D1 branes allows one to consider $k$ independent minimal couplings (3.19) to the $b$ field. This might be worth studying because it could 


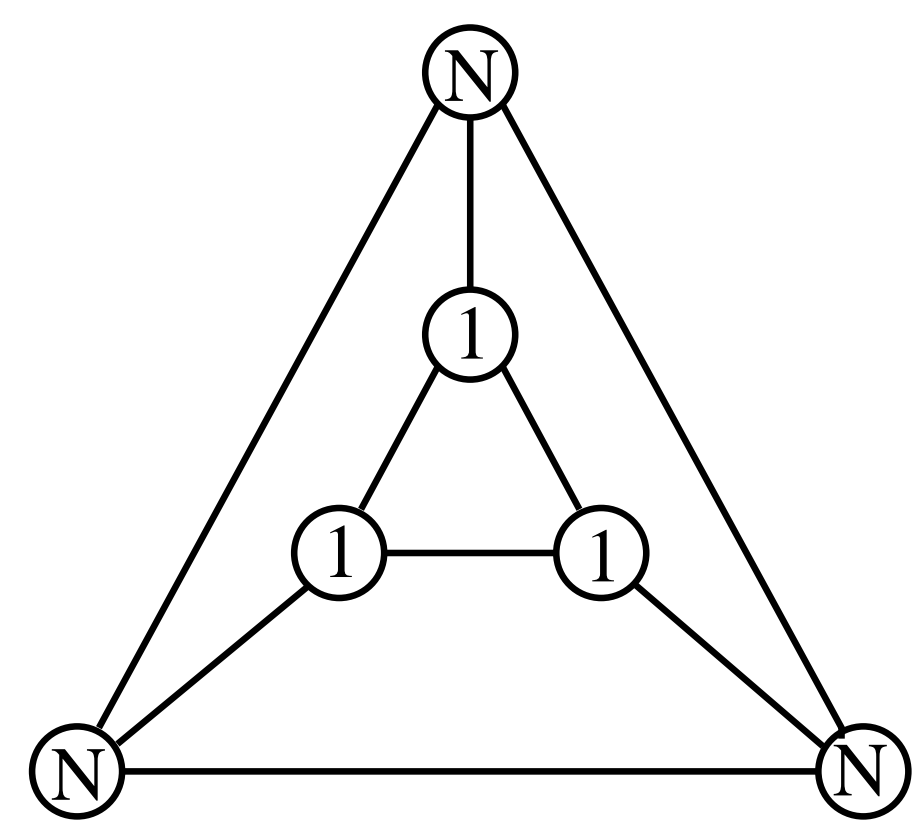

Figure 3: The quiver for the D1-D5 brane system in the case $k=3$.

provide a mechanism to generate $k$ independent coupling constants, one at each node. Since in the usual case the moduli space of couplings is related to twisted sectors, it will be valuable to clarify this issue further by studying the closed sector of the B-model and check whether it contains twisted states.

\section{Explicit examples of orbifolds}

\subsection{An $\mathcal{N}=1$ orbifold}

We start by considering the case $\Gamma \in Z_{k} \subset S U(3)_{R}$. Then $S U(4)_{R}$ is broken into $U(1)_{R}$, yielding $\mathcal{N}=1$. We consider a particularly simple example, in which $k=3$ and $a_{A}=$ $(1,1,1,0)$, see (3.7). The gauge group is decomposed into $[U(N)]^{3}$ and the corresponding quiver diagram is depicted in Figure 4.

The gauge sector contains three $\mathcal{N}=1$ vector multiplets

$$
A_{\mathbf{i}}, \quad G_{\mathbf{i}}, \quad \lambda_{\mathbf{i}}, \quad \tilde{\lambda}_{\mathbf{i}}
$$

where $A_{\mathbf{i}} \equiv A_{J \mathbf{i}}^{I \mathbf{i}}, G_{\mathbf{i}} \equiv G_{J \mathbf{i}}^{I \mathbf{i}}, \lambda_{\mathbf{i}}^{a} \equiv\left(\chi_{4}\right)_{J \mathbf{i}}^{I \mathbf{i}}, \tilde{\lambda}_{\mathbf{i}}^{a} \equiv\left(\tilde{\chi}_{4}\right)_{J \mathbf{i}}^{I \mathbf{i}}$. The index $\mathbf{i}=1,2,3$ labels the nodes of the quiver.

On the other hand, the matter sector consists of three $\mathcal{N}=1$ chiral multiplets for each pair of nodes

$$
q_{\mathbf{i}, \mathbf{i}+\mathbf{1}}^{\mu}, \quad \tilde{q}_{\mathbf{i}+\mathbf{1}, \mathbf{i}}^{\mu}, \quad \phi_{\mathbf{i}, \mathbf{i}+\mathbf{1}}^{\mu}, \quad \tilde{\phi}_{\mathbf{i}+\mathbf{1}, \mathbf{i}}^{\mu}
$$




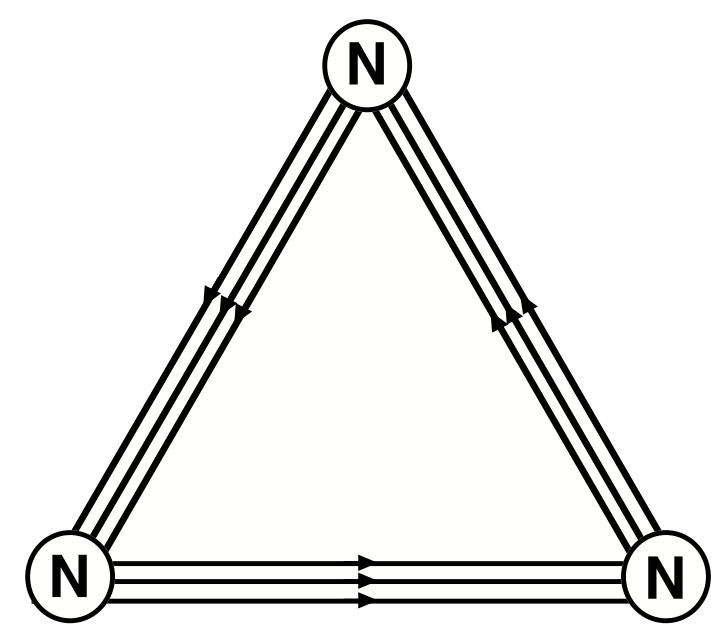

Figure 4: The $\mathcal{N}=1$ quiver for $k=3$ and $a_{A}=(1,1,1,0)$.

where now the index $\mu$ runs from 1 to 3 . Here a subscript $\mathbf{i}, \mathbf{j}$ indicates that the field has fundamental index in the $\mathbf{i}$-th node and anti-fundamental in the $\mathbf{j}$-th node. The quarks $q_{\mathbf{i}, \mathbf{i}+\mathbf{1}}^{\mu}$ and the anti-quarks $\tilde{q}_{\mathbf{i}+\mathbf{1}, \mathbf{i}}^{\mu}$ come from $\left(\chi^{\mu}\right)_{J, \mathbf{i}+\mathbf{1}}^{I \mathbf{i}}$ and $\left(\tilde{\chi}^{\mu}\right)_{J \mathbf{i}}^{I, \mathbf{i}+\mathbf{1}}$. The scalars $\phi_{\mathbf{i}, \mathbf{i}+\mathbf{1}}^{\mu}$ and $\tilde{\phi}_{\mathbf{i}+\mathbf{1}, \mathbf{i}}^{\mu}$ come from $\left(\phi_{\mu 4}\right)_{J, \mathbf{i}+\mathbf{1}}^{I \mathbf{i}}$ and $\epsilon^{\mu \nu \rho}\left(\phi_{\nu \rho}\right)_{J \mathbf{i}}^{I, \mathbf{i}+\mathbf{1}}$. The gauge theory with this field content is superconformal [16] [17].

In terms of these fields the action (3.6) becomes

$$
\begin{aligned}
\mathcal{S}=\sum_{\mathbf{i}=1}^{3} \int_{C P^{3}} \Omega^{\prime} & \wedge \operatorname{Tr}\left[G_{\mathbf{i}} \wedge\left(\bar{\partial} A_{\mathbf{i}}+A_{\mathbf{i}} \wedge A_{\mathbf{i}}\right)+\tilde{\lambda}_{\mathbf{i}} \wedge \bar{D}_{\mathbf{i}} \lambda_{\mathbf{i}}\right. \\
& +\tilde{q}_{\mu \mathbf{i}+\mathbf{1}, \mathbf{i}} \wedge \bar{D}_{\mathbf{i}} q_{\mathbf{i}, \mathbf{i}+\mathbf{1}}^{\mu}+\tilde{\phi}_{\mu \mathbf{i}+\mathbf{1}, \mathbf{i}} \wedge \bar{D}_{\mathbf{i}} \phi_{\mathbf{i}, \mathbf{i}+\mathbf{1}}^{\mu} \\
& \left.+\epsilon_{\mu \nu \rho} q_{\mathbf{i}, \mathbf{i}+\mathbf{1}}^{\mu} \wedge q_{\mathbf{i}+\mathbf{1}, \mathbf{i}+\mathbf{2}}^{\nu} \wedge \phi_{\mathbf{i}+\mathbf{2}, \mathbf{i}}^{\rho}+\lambda_{\mathbf{i}} \wedge q_{\mathbf{i}, \mathbf{i}+\mathbf{1}}^{\mu} \wedge \tilde{\phi}_{\mu \mathbf{i}+\mathbf{1}, \mathbf{i}}\right]
\end{aligned}
$$

The interaction term (3.15) becomes after the orbifold projection

$$
\begin{aligned}
\Delta I_{D 1-D 5} & =\int_{D 1} \operatorname{Tr} J \mathcal{A}=\int_{D 1} J_{J \mathbf{j}}^{I \mathbf{i}} \mathcal{A}_{I \mathbf{i}}^{J \mathbf{j}} \rightarrow \\
& \rightarrow \sum_{\mathbf{i}=1}^{3} \int_{D 1} \operatorname{Tr}\left[J_{\mathbf{i}} A_{\mathbf{i}}+\psi^{4} J_{\mathbf{i}} \lambda_{\mathbf{i}}+\psi^{\mu} J_{\mathbf{i}+\mathbf{1}, \mathbf{i}} q_{\mu \mathbf{i}, \mathbf{i}+\mathbf{1}}\right. \\
& +\frac{1}{2} \epsilon_{\mu \nu \rho} \psi^{\mu} \psi^{\nu} J_{\mathbf{i}, \mathbf{i}+\mathbf{1}} \tilde{\phi}_{\mathbf{i}+\mathbf{1}, \mathbf{i}}^{\rho}+\psi^{\mu} \psi^{4} J_{\mathbf{i}+\mathbf{1}, \mathbf{i}} \phi_{\mu \mathbf{i}, \mathbf{i}+\mathbf{1}} \\
& \left.+\frac{1}{3 !} \epsilon_{\mu \nu \rho} \psi^{\mu} \psi^{\nu} \psi^{\rho} J_{\mathbf{i}} \tilde{\lambda}_{\mathbf{i}}+\frac{1}{2} \epsilon_{\mu \nu \rho} \psi^{\mu} \psi^{\nu} \psi^{4} J_{\mathbf{i}, \mathbf{i}+\mathbf{1}} \tilde{q}_{\mathbf{i}+\mathbf{1}, \mathbf{i}}^{\rho}+\psi^{1} \psi^{2} \psi^{3} \psi^{4} J_{\mathbf{i}} G_{\mathbf{i}}\right]
\end{aligned}
$$

with $J_{\mathbf{i}} \equiv J_{I \mathbf{i}}^{J \mathbf{i}}=\alpha^{J \mathbf{i}} \beta_{I \mathbf{i}}$ and $J_{\mathbf{i}, \mathbf{i}+\mathbf{1}} \equiv J_{I \mathbf{i}}^{J, \mathbf{i}+\mathbf{1}}=\alpha^{J, \mathbf{i}+\mathbf{1}} \beta_{I \mathbf{i}}$. A convenient way to keep track of the group theory factors is to use a double line notation, where one assigns a different type of oriented line to the fundamental index of each node. For instance, an adjoint 


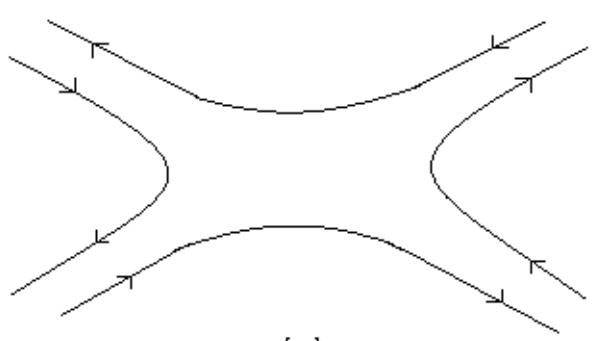

(a)

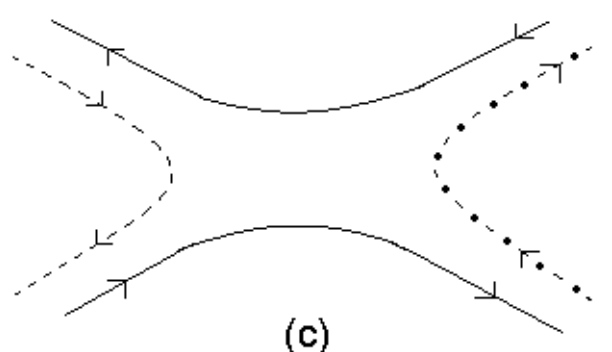

(c)

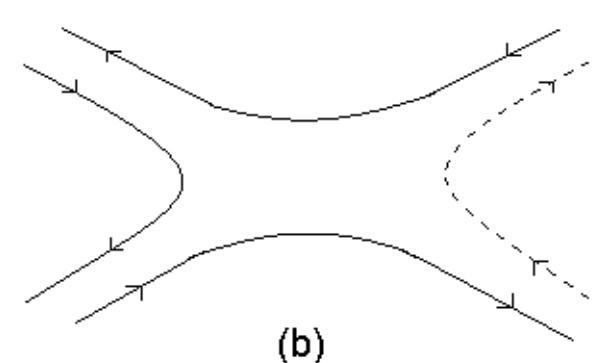

(b)

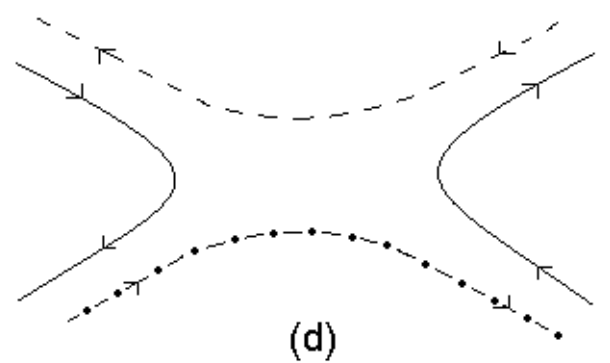

Figure 5: The double line notation: (a) scattering of four adjoint fields belonging to the same node; (b) scattering of two adjoint and two bi-fundamental fields; (c) scattering of four bi-fundamental fields with intermediate adjoint field; (d) same as in (c) but with intermediate bi-fundamental field.

field is represented by two lines of the same type and opposite orientation, while a bifundamental field has two lines of different type and opposite orienation. For example, in the $\mathcal{N}=1$ case discussed here there are three types of lines corresponding to the three nodes of the quiver in Figure 4 . Some examples are shown in Figure 5.

\subsubsection{MHV Amplitudes for the $\mathcal{N}=1$ orbifold}

In order to calculate MHV amplitudes in these theories we need to follow the general prescription given in [1] and 27]. This prescription is applicable also in this case since we do not allow the $k$ D1 branes to move independently, as already discussed. Saturation of the fermionic degrees of freedom requires eight $\theta$ 's. We consequently have as many different MHV amplitudes, as there are possible products of terms from the superfield expansion giving eight $\theta$ 's. The denominator of these analytic amplitudes is provided by the current correlation functions. The latters also provide the appropriate group structure. Note that here we should be a little bit more careful than usual, since part of the gauge theory trace is implicit in the summation of the indices $\mathbf{i}$ which belong to the fundamental representation. We should therefore make sure that we consider only meaningful products of currents, that correspond to single trace terms for each MHV analytic amplitude, for 
instance products like $J_{\mathbf{i}} J_{\mathbf{i}, \mathbf{i}+\mathbf{1}} J_{\mathbf{i}+\mathbf{1}, \mathbf{i}} J_{\mathbf{i}}$ for a four point amplitude of the form $(\lambda \tilde{q} q \tilde{\lambda})$. The possible group theory contractions are easily estabilished by drawing the diagrams in double-line notation.

It is now straightforward to proceed to the computation of specific amplitudes of interest. One could rewrite the $\mathcal{N}=4$ superfield expansion (3.4) in $S U(3) \times U(1)_{R}$ notation which is manifestly $\mathcal{N}=1$ invariant. Recalling that the momentum structure of the amplitudes is solely determined by the form of this expansion, we deduce that analytic amplitudes in the $\mathcal{N}=1$ orbifold theory bear an identical spinor product structure to the ones of $\mathcal{N}=4 \mathrm{SYM}$ in $S U(3) \times U(1)_{R}$ notation. This is in complete accordance with field theoretical considerations, since the Lagrangian description of these theories is identical apart from their group structure. We will make this point clearer with several examples. Note, however, that in what follows we will omit group indices ${ }^{12}$ and coupling constants, since we stay at the point in the moduli space where all the gauge couplings are equal.

Example 1: Amplitudes $(A \ldots A G G),(A \ldots A G \lambda \tilde{\lambda})$, and $(A \ldots A G q \tilde{q})$ These amplitudes have been extensively considered in the literature (for instance, see [28]). As a first trivial check of the above, we compute the standard four gluon amplitude $\left(A_{\mathbf{i}} A_{\mathbf{i}} G_{\mathbf{i}} G_{\mathbf{i}}\right)$, with $\mathbf{i}=1,2,3$. Following the usual prescription, and using (3.4), we have

$$
A^{(A A G G)}=\int d^{8} \theta\left(\psi_{3}^{1} \psi_{3}^{2} \psi_{3}^{3} \psi_{3}^{4}\right)\left(\psi_{4}^{1} \psi_{4}^{2} \psi_{4}^{3} \psi_{4}^{4}\right) \frac{1}{\langle 12\rangle\langle 23\rangle\langle 34\rangle\langle 41\rangle}=\frac{\langle 34\rangle^{4}}{\langle 12\rangle\langle 23\rangle\langle 34\rangle\langle 41\rangle} .
$$

This is the familiar formula for MHV scattering in $\mathcal{N}=4$ SYM. In the same way, one can compute amplitudes of the type $(A \ldots A G G),(A \ldots A G \lambda \tilde{\lambda}),(A \ldots A G q \tilde{q})$, and $(A \ldots A \lambda \tilde{\lambda} q \tilde{q})$.

Example 2: Amplitudes $(q q \tilde{q} \tilde{q})$ and $(\lambda q \tilde{q} \tilde{\lambda})$ These amplitudes have, as previously mentioned, the same spinor product structure $\mathcal{N}=4 \mathrm{SYM}$ has. Yet, they are far more interesting cases to study. The reason is that they consist of two subamplitudes, shown in the case $(q q \tilde{q} \tilde{q})$ in Figure 6. They depend on both gluon and scalar particle exchange. Let us now concentrate on $\left(q_{\mathbf{i}-\mathbf{1}, \mathbf{i}}^{\rho} q_{\mathbf{i}, \mathbf{i}+\mathbf{1}}^{\sigma} \tilde{q}_{\mathbf{i}+\mathbf{1}, \mathbf{i}}^{\kappa} \tilde{q}_{\mathbf{i}, \mathbf{i}-\mathbf{1}}^{\lambda}\right)$. The other case $(\lambda q \tilde{q} \tilde{\lambda})$ can be computed in a similar manner. The two subamplitudes in Figure 6 correspond in double line notation to diagrams (d) and (c) in Figure 5

$$
A^{(q q \tilde{q} \tilde{q})}=\int d^{8} \theta \frac{1}{4} \psi_{1}^{\rho} \psi_{2}^{\sigma}\left(\psi_{3}^{4} \psi_{3}^{\mu} \psi_{3}^{\nu}\right)\left(\psi_{4}^{4} \psi_{4}^{\pi} \psi_{4}^{\tau}\right) \epsilon_{\mu \nu \kappa} \epsilon_{\pi \tau \lambda} \frac{1}{\langle 12\rangle\langle 23\rangle\langle 34\rangle\langle 41\rangle} .
$$

Integration over the $\theta^{4}$ is straightforward and yields $\langle 34\rangle$. Then, we must sum over all possible contractions of momenta upon integration over the fermionic part of the space.

\footnotetext{
${ }^{12}$ As usual we strip out the gauge group theory factor.
} 
There are three distinct ones

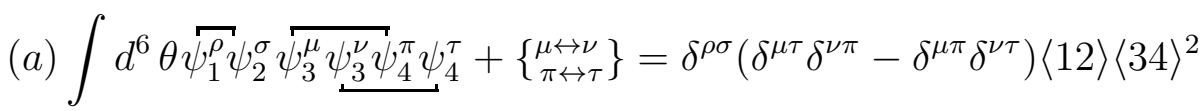

(b) $\int d^{6} \theta \overline{\psi_{1}^{\rho} \psi_{2}^{\sigma} \psi_{3}^{\mu} \psi_{3}^{\nu} \psi_{4}^{\pi}} \psi_{4}^{\tau}+\left\{\begin{array}{c}\mu \leftrightarrow \nu \\ \pi \leftrightarrow \tau\end{array}\right\}=\left(\delta^{\rho \sigma}\left(\delta^{\mu \pi} \delta^{\nu \tau}-\delta^{\mu \tau} \delta^{\nu \pi}\right)-\epsilon^{\rho \mu \nu} \epsilon^{\sigma \pi \tau}\right)\langle 23\rangle\langle 34\rangle\langle 41\rangle(4.7)$

(c) $\int d^{6} \theta \overline{\psi_{1}^{\rho} \underline{\psi}_{2}^{\sigma} \psi_{3}^{\mu}} \bar{\psi}_{3}^{\nu} \psi_{4}^{\pi} \psi_{4}^{\tau}+\left\{\begin{array}{c}\mu \leftrightarrow \nu \\ \pi \leftrightarrow \tau\end{array}\right\}=\left(\delta^{\rho \sigma}\left(\delta^{\mu \pi} \delta^{\nu \tau}-\delta^{\mu \tau} \delta^{\nu \pi}\right)-\epsilon^{\rho \pi \tau} \epsilon^{\sigma \mu \nu}\right)$

The three spinor product structures in (4.7), are related through the Schouten Identity

$$
\langle p q\rangle\langle r s\rangle+\langle q r\rangle\langle p s\rangle+\langle r p\rangle\langle q s\rangle=0
$$

Use of this identity reveals the two independent structures that we were expecting. Explicitly, we have

$$
\int d^{6} \theta \psi_{1}^{\rho} \psi_{2}^{\sigma} \psi_{3}^{\mu} \psi_{3}^{\nu} \psi_{4}^{\pi} \psi_{4}^{\tau}=-\epsilon^{\rho \pi \tau} \epsilon^{\sigma \mu \nu}\langle 12\rangle\langle 34\rangle^{2}+\left(\epsilon^{\rho \pi \tau} \epsilon^{\sigma \mu \nu}-\epsilon^{\rho \mu \nu} \epsilon^{\sigma \pi \tau}\right)\langle 23\rangle\langle 34\rangle\langle 41\rangle .
$$

Inserting (4.9) into (4.6), we obtain

$$
A^{(q q \tilde{q} \tilde{q})}=-\delta^{\rho}{ }_{\lambda} \delta^{\sigma}{ }_{\kappa} \frac{\langle 34\rangle^{2}}{\langle 23\rangle\langle 41\rangle}-\epsilon^{\rho \sigma \tau} \epsilon_{\tau \kappa \lambda} \frac{\langle 34\rangle}{\langle 12\rangle} .
$$

It is easy to see that this result is in agreement with the field theory predictions. An important remark is now in order. As we can also see in Figure 6, there are two types of contributions to this scattering process. One of them comes from a Yukawa type interaction term while the other comes from the usual matter-gluon interaction. In general these two interaction terms would be weighted with the appropriate independent coupling constant. It would be interesting to check if the consistency of the twistor method constraints the couplings to be in the conformal region of the moduli space. Amplitudes like the one considered in this example might provide some insight on how to move away from the point in the moduli space where all the couplings are equal.

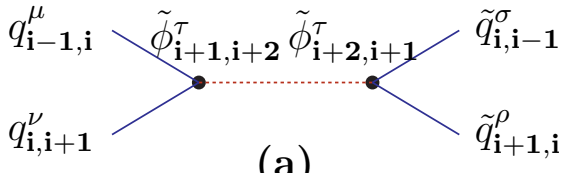

(a)

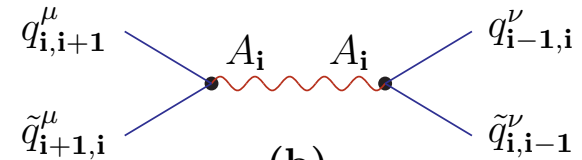

(b)

Figure 6: The two Feynman diagrams that contribute to tree-level $(q q \tilde{q} \tilde{q})$ scattering. 


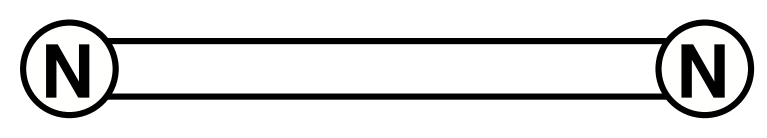

Figure 7: The $\mathcal{N}=2$ quiver for $k=2$ and $a_{A}=(1,1,0,0)$.

\subsection{An $\mathcal{N}=2$ orbifold}

We now move on to the case in which $\Gamma \in Z_{k} \subset S U(2)_{R}$. This breaks $S U(4)_{R} \rightarrow S U(2)_{R}$, thus giving $\mathcal{N}=2$. For simplicity, we investigate the particular choice of $k=2$ and $a_{A}=(1,1,0,0)$, see (3.7). The fields surviving the orbifold projection are organized into two $\mathcal{N}=2$ vector multiplets and two hypermultiplets. The resulting gauge group is $U(N) \times U(N)$. The associated quiver diagram has two nodes and two links and is given in Figure 7 . As in the previous $\mathcal{N}=1$ case, this theory is superconformal.

The field content of the gauge sector is

$$
A_{\mathbf{i}}, \quad G_{\mathbf{i}}, \quad \lambda_{\mathbf{i}}^{a}, \quad \tilde{\lambda}_{\mathbf{i}}^{a}, \quad \phi_{\mathbf{i}}^{m}
$$

where $A_{\mathbf{i}} \equiv A_{J \mathbf{i}}^{I \mathbf{i}}, G_{\mathbf{i}} \equiv G_{J \mathbf{i}}^{I \mathbf{i}}, \lambda_{\mathbf{i}}^{a} \equiv\left(\chi_{3,4}\right)^{I \mathbf{i}}, \tilde{\lambda}_{\mathbf{i}}^{a} \equiv\left(\tilde{\chi}_{3,4}\right)_{J \mathbf{i}}^{I \mathbf{i}}$, and $\phi_{\mathbf{i}}^{m} \equiv\left(\phi_{12,34}\right)^{I \mathbf{i}}$. The nodes of the quiver are labelled by $\mathbf{i}=1,2$. The index $a$ is the $S U(2)_{R}$ index, whereas $m$ labels the two real components of the complex scalar field. The matter sector has two bifundamental hypermultiplets

$$
q_{\mathbf{i}, \mathbf{i}+\mathbf{1}}^{\mu}, \quad \tilde{q}_{\mathbf{i}+\mathbf{1}, \mathbf{i}}^{\mu}, \quad H_{\mathbf{i}, \mathbf{i}+\mathbf{1}}^{\mu}, \quad \tilde{H}_{\mathbf{i}+\mathbf{1}, \mathbf{i}}^{\mu}
$$

with the index $\mu=1,2$ labels the hypermultiplets. The quarks $q_{\mathbf{i}, \mathbf{i}+\mathbf{1}}^{\mu}$ are given by $\left(\chi^{1,2}\right)_{J, \mathbf{i}+\mathbf{1}}^{I \mathbf{i}}$ and the anti-quarks $\tilde{q}_{\mathbf{i}+\mathbf{1}, \mathbf{i}}^{\mu}$ by $\left(\tilde{\chi}^{1,2}\right)_{J \mathbf{i}}^{I, \mathbf{i}+\mathbf{1}}$. The four scalars $H^{\mu}$ and $\tilde{H}^{\mu}$ come from $\phi_{13}, \phi_{14}, \phi_{23}$, and $\phi_{24}$.

The projected action and the D1-D5 interaction term can be obtained in similarly to the previous $\mathcal{N}=1$ case.

\subsubsection{MHV Amplitudes for the $\mathcal{N}=2$ orbifold}

Example 1: Amplitudes like $(A \ldots A G G),(A \ldots A G, \lambda \tilde{\lambda})$, and $(A \ldots A G q \tilde{q})$ We consider the scattering process between the following particles $\left(A A G \lambda^{a} \tilde{\lambda}^{c}\right)$. According to the twistor string method, we should compute

$$
A^{(A A G \lambda \tilde{\lambda})}=\int d^{8} \theta \psi_{3}^{1} \psi_{3}^{2} \psi_{3}^{3} \psi_{3}^{4} \psi_{4}^{a} \psi_{5}^{1} \psi_{5}^{2} \psi_{5}^{b} \epsilon_{b c} \frac{1}{\langle 12\rangle\langle 23\rangle\langle 34\rangle\langle 41\rangle} .
$$

There are only two possible contractions between the different $\psi$ 's, which yield

$$
\begin{aligned}
A^{(A A G \lambda \tilde{\lambda})} & =\left(\delta^{3 b} \delta^{4 a}-\delta^{3 a} \delta^{4 b}\right) \epsilon_{b c} \frac{\langle 35\rangle^{3}\langle 34\rangle}{\langle 12\rangle\langle 23\rangle\langle 34\rangle\langle 45\rangle\langle 51\rangle} \\
& =\delta^{a c}\left(\delta^{4 c}-\delta^{3 c}\right) \frac{\langle 35\rangle^{3}}{\langle 12\rangle\langle 23\rangle\langle 45\rangle\langle 51\rangle} .
\end{aligned}
$$


As we immediately see, we recovered the familiar result.

Example 2: Amplitude $(\lambda q \tilde{q} \tilde{\lambda})$ We will now apply the same method in order to compute the scattering amplitude $\left(\lambda^{a} q^{\mu} \tilde{q}^{\rho} \tilde{\lambda}^{c}\right)$ between a gluino-antigluino pair and a quark-antiquark one. To this end, we need to calculate the following integral

$$
A^{(\lambda q \tilde{q} \tilde{\lambda})}=\int d^{8} \theta \psi_{1}^{a} \psi_{2}^{\mu}\left(\psi_{3}^{3} \psi_{3}^{4} \psi_{3}^{\nu}\right)\left(\psi_{4}^{1} \psi_{4}^{2} \psi_{4}^{b}\right) \epsilon_{\nu \rho} \epsilon_{b c} \frac{1}{\langle 12\rangle\langle 23\rangle\langle 34\rangle\langle 41\rangle}
$$

where $a, b, c=3,4$ and $\mu, \nu, \rho=1,2$. To perform the integration we need to sum over all the possible contractions between the fermionic coordinates of supertwistor space. In this example we can split the fermions into two groups, with no contractions among fermions belonging to different groups. In each group, fermions can be contracted in two different ways

$$
\begin{aligned}
& \text { (a) } \int d^{4} \theta \bar{\psi}_{1}^{a} \psi_{3}^{3} \psi_{3}^{4} \psi_{4}^{b}=\delta^{a 3} \delta^{4 b}\langle 13\rangle\langle 34\rangle \\
& \text { (b) } \int d^{4} \theta \sqrt{\psi_{1}^{a} \psi_{3}^{3} \psi_{3}^{4} \psi_{4}^{b}}=-\delta^{a 4} \delta^{b 3}\langle 13\rangle\langle 34\rangle
\end{aligned}
$$

and

$$
\begin{aligned}
& \text { (a) } \int d^{4} \theta \overline{\psi_{2}^{\mu} \psi_{3}^{\nu} \psi_{4}^{1} \psi_{4}^{2}}=\delta^{\mu 2} \delta^{\nu 1}\langle 24\rangle\langle 34\rangle \\
& \text { (b) } \int d^{4} \theta \overline{\psi_{2}^{\mu} \underline{\psi}_{3}^{\nu} \psi_{4}^{1} \psi_{4}^{2}}=-\delta^{\mu 1} \delta^{\nu 2}\langle 24\rangle\langle 34\rangle .
\end{aligned}
$$

We then substitute (4.16) and (4.17) into (4.15) and use the Schouten Identity to obtain

$$
A^{(\lambda q \tilde{q} \tilde{\lambda})}=\delta^{a c} \delta^{\mu \rho}\left(\delta^{\mu 2}-\delta^{\mu 1}\right)\left(\delta^{a 3}-\delta^{a 4}\right)\left(\frac{\langle 34\rangle^{2}}{\langle 23\rangle\langle 41\rangle}-\frac{\langle 34\rangle}{\langle 12\rangle}\right) .
$$

We see from the form of the result that there are exactly two distinct spinor product structures. They reflect contributions to the scattering process from two different types of interactions: the former is the standard quark-gluon interaction and the latter is of Yukawa type. Figure 8 shows the corresponding Feynman diagramms. This is in accordance with the usual field theory calculations.

Other amplitudes, with quarks or scalars as external particles, can be computed in a similar fashion. They usually retain the feature of receiving contributions from multiple interaction processes/vertices.

\section{Conclusion}

In this paper we have investigated $Z_{k}$ fermionic orbifolds of the topological B-model on $C P^{(3 \mid 4)}$ to reduce the amount of supersymmetry of the dual $\mathcal{N}=4$ Super Yang-Mills 


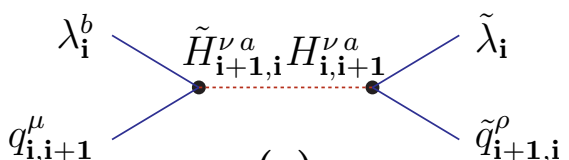

(a)

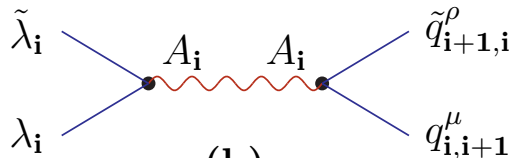

(b)

Figure 8: The two Feynman diagrams that contribute to tree-level $(\lambda q \tilde{q} \tilde{\lambda})$ scattering.

theory. This is possible since the Fermi-Fermi part of the $P S U(4 \mid 4)$ isometry group of $C P^{(3 \mid 4)}$ is precisely the $S U(4)_{R} R$-symmetry. We have discussed how the projection acts on both the D5 and the D1 branes. As examples we have considered $\mathcal{N}=1$ and $\mathcal{N}=2$ orbifolds and obtained the corresponding quiver theories. Several amplitudes have been computed and shown to agree with the field theory results.

Throughout the paper we have worked only at the point in the moduli space where all gauge couplings constants are equal. It would be interesting to study the full moduli space of superconformal couplings and understand its interpretation in twistor string theory. Some indications on the origin of these moduli have been given in discussing the action of the orbifold on the D1 brane sector. Since these moduli are usually interpreted as coming from twisted fields it would be worth studying the closed string sector and identify the twisted states. The fermionic orbifold does not have an obvious geometrical meaning. The study of the twisted sector may be useful to shed some light on the geometrical interpretation of the orbifold.

Acknowledgements It is a pleasure to thank Martin Roček and Albert Schwarz for useful discussions and comments. We would also like to express our gratitude to the participants and the organizers of the second Simons Workshop in Mathematics and Physics at Stony Brook for creating a stimulating environment from which we benefited greatly. We acknowledge partial financial support through NSF award PHY-0354776.

Note added in proof After completion of our computations, while writing up the results, we became aware of [29], which has overlap with our work.

\section{References}

[1] E. Witten, "Perturbative gauge theory as a string theory in twistor space," arXiv:hep-th/0312171.

[2] R. Roiban, M. Spradlin and A. Volovich, "A googly amplitude from the B-model in twistor space," JHEP 0404, 012 (2004) arXiv:hep-th/0402016; R. Roiban and 
A. Volovich, "All conjugate-maximal-helicity-violating amplitudes from topological open string theory in twistor space," arXiv:hep-th/0402121; R. Roiban, M. Spradlin and A. Volovich, "On the tree-level S-matrix of Yang-Mills theory," Phys. Rev. D 70, 026009 (2004) arXiv:hep-th/0403190.

[3] F. Cachazo, P. Svrcek and E. Witten, "MHV vertices and tree amplitudes in gauge theory," JHEP 0409, 006 (2004) arXiv:hep-th/0403047.

[4] C. J. Zhu, "The googly amplitudes in gauge theory," JHEP 0404, 032 (2004) arXiv:hep-th/0403115; G. Georgiou and V. V. Khoze, "Tree amplitudes in gauge theory as scalar MHV diagrams," JHEP 0405, 070 (2004) arXiv:hep-th/0404072; J. B. $\mathrm{Wu}$ and $\mathrm{C}$. J. Zhu, "MHV vertices and scattering amplitudes in gauge theory," JHEP 0407, 032 (2004) arXiv:hep-th/0406085; I. Bena, Z. Bern and D. A. Kosower, "Twistor-space recursive formulation of gauge theory amplitudes," arXiv:hep-th/0406133; J. B. Wu and C. J. Zhu, "MHV vertices and fermionic scattering amplitudes in gauge theory with quarks and gluinos," JHEP 0409, 063 (2004) arXiv:hep-th/0406146; D. A. Kosower, "Next-to-maximal helicity violating amplitudes in gauge theory," arXiv:hep-th/0406175; G. Georgiou, E. W. N. Glover and V. V. Khoze, "Non-MHV tree amplitudes in gauge theory," JHEP 0407, 048 (2004) arXiv:hep-th/0407027; Y. Abe, V. P. Nair and M. I. Park, "Multigluon amplitudes, $\mathrm{N}=4$ constraints and the WZW model," arXiv:hep-th/0408191; L. J. Dixon, E. W. N. Glover and V. V. Khoze, "MHV rules for Higgs plus multi-gluon amplitudes," arXiv:hep-th/0411092;

[5] V. V. Khoze, "Gauge theory amplitudes, scalar graphs and twistor space," arXiv:hep-th/0408233.

[6] S. Gukov, L. Motl and A. Neitzke, "Equivalence of twistor prescriptions for super Yang-Mills," arXiv:hep-th/0404085.

[7] F. Cachazo, P. Svrcek and E. Witten, "Twistor space structure of one-loop amplitudes in gauge theory," JHEP 0410, 074 (2004) arXiv:hep-th/0406177; A. Brandhuber, B. Spence and G. Travaglini, "One-loop gauge theory amplitudes in N $=4$ super Yang-Mills from MHV vertices," arXiv:hep-th/0407214; F. Cachazo, P. Svrcek and E. Witten, "Gauge theory amplitudes in twistor space and holomorphic anomaly," JHEP 0410, 077 (2004) arXiv:hep-th/0409245; M. x. Luo and C. k. Wen, "Oneloop maximal helicity violating amplitudes in $\mathrm{N}=4$ super Yang-Mills theories," arXiv:hep-th/0410045; I. Bena, Z. Bern, D. A. Kosower and R. Roiban, "Loops in twistor space," arXiv:hep-th/0410054; F. Cachazo, "Holomorphic anomaly of unitarity cuts and one-loop gauge theory amplitudes," arXiv:hep-th/0410077; 
M. x. Luo and C. k. Wen, "Systematics of one-loop scattering amplitudes in N = 4 super Yang-Mills theories," arXiv:hep-th/0410118; R. Britto, F. Cachazo and B. Feng, "Computing one-loop amplitudes from the holomorphic anomaly of unitarity cuts," arXiv:hep-th/0410179; Z. Bern, V. Del Duca, L. J. Dixon and D. A. Kosower, "All non-maximally-helicity-violating one-loop seven-gluon amplitudes in $\mathrm{N}=4$ superYang-Mills theory," arXiv:hep-th/0410224; C. Quigley and M. Rozali, "One-loop MHV amplitudes in supersymmetric gauge theories," arXiv:hep-th/0410278; J. Bedford, A. Brandhuber, B. Spence and G. Travaglini, "A twistor approach to one-loop amplitudes in $\mathrm{N}=1$ supersymmetric Yang-Mills theory," arXiv:hep-th/0410280; S. J. Bidder, N. E. J. Bjerrum-Bohr, L. J. Dixon and D. C. Dunbar, "N= 1 supersymmetric one-loop amplitudes and the holomorphic anomaly of unitarity cuts," arXiv:hep-th/0410296; R. Britto, F. Cachazo and B. Feng, "Coplanarity in twistor space of $\mathrm{N}=4$ next-to-MHV one-loop amplitude coefficients," arXiv:hep-th/0411107.

[8] N. Berkovits, "An alternative string theory in twistor space for $\mathrm{N}=4$ super-YangMills," Phys. Rev. Lett. 93, 011601 (2004) arXiv:hep-th/0402045; N. Berkovits and L. Motl, "Cubic twistorial string field theory," JHEP 0404, 056 (2004) arXiv:hep-th/0403187.

[9] A. Neitzke and C. Vafa, "N=2 strings and the twistorial Calabi-Yau," arXiv:hep-th/0402128; N. Nekrasov, H. Ooguri and C. Vafa, "S-duality and topological strings," JHEP 0410, 009 (2004) arXiv:hep-th/0403167; M. Aganagic and C. Vafa, "Mirror symmetry and supermanifolds," arXiv:hep-th/0403192.

[10] W. Siegel, "Untwisting the twistor superstring," arXiv:hep-th/0404255.

[11] N. Berkovits and E. Witten, "Conformal supergravity in twistor-string theory," JHEP 0408, 009 (2004) arXiv:hep-th/0406051.

[12] C. h. Ahn, " $\mathrm{N}=1$ conformal supergravity and twistor-string theory," JHEP $\mathbf{0 4 1 0}$, 064 (2004) arXiv:hep-th/0409195.

[13] S. Giombi, R. Ricci, D. Robles-Llana and D. Trancanelli, "A note on twistor gravity amplitudes," JHEP 0407, 059 (2004) arXiv:hep-th/0405086.

[14] A. D. Popov and C. Saemann, "On supertwistors, the Penrose-Ward transform and N = 4 super Yang-Mills theory," arXiv:hep-th/0405123; O. Lechtenfeld and A. D. Popov, "Supertwistors and cubic string field theory for open $\mathrm{N}=2$ strings," Phys. Lett. B 598, 113 (2004) arXiv:hep-th/0406179; A. D. Popov and M. Wolf, "Topological B-model on weighted projective spaces and self-dual models in four 
dimensions," JHEP 0409, 007 (2004) arXiv:hep-th/0406224; C. h. Ahn, "Mirror symmetry of Calabi-Yau supermanifolds," arXiv:hep-th/0407009; A. Sinkovics and E. Verlinde, "A six dimensional view on twistors," arXiv:hep-th/0410014; C. Saemann, "The topological B-model on fattened complex manifolds and subsectors of $\mathrm{N}$ $=4$ self-dual Yang-Mills theory," arXiv:hep-th/0410292.

[15] M. Kulaxizi and K. Zoubos, "Marginal deformations of N =4 SYM from open / closed twistor strings," arXiv:hep-th/0410122.

[16] S. Kachru and E. Silverstein, "4d conformal theories and strings on orbifolds," Phys. Rev. Lett. 80, 4855 (1998) arXiv:hep-th/9802183.

[17] A. E. Lawrence, N. Nekrasov and C. Vafa, "On conformal field theories in four dimensions," Nucl. Phys. B 533, 199 (1998) arXiv:hep-th/9803015.

[18] M. R. Douglas and G. W. Moore, "D-branes, Quivers, and ALE Instantons," arXiv:hep-th/9603167; M. R. Douglas, B. R. Greene and D. R. Morrison, "Orbifold resolution by D-branes," Nucl. Phys. B 506, 84 (1997) arXiv:hep-th/9704151.

[19] F. Cachazo, S. Katz and C. Vafa, "Geometric transitions and N = 1 quiver theories," arXiv:hep-th/0108120; F. Cachazo, B. Fiol, K. A. Intriligator, S. Katz and C. Vafa, "A geometric unification of dualities," Nucl. Phys. B 628, 3 (2002) arXiv:hep-th/0110028.

[20] E. Witten, Nucl. Phys. B 500, 3 (1997) arXiv:hep-th/9703166.

[21] S. Katz, P. Mayr and C. Vafa, "Mirror symmetry and exact solution of 4D N = 2 gauge theories. I," Adv. Theor. Math. Phys. 1, 53 (1998) arXiv:hep-th/9706110.

[22] R. Penrose, "Twistor Algebra," J. Math. Phys. 8, 345 (1967).

[23] W. Siegel, "N=2, N=4 string theory is selfdual N=4 Yang-Mills theory," Phys. Rev. D 46, 3235 (1992); "Selfdual N=8 supergravity as closed $\mathrm{N}=2(\mathrm{~N}=4)$ strings," Phys. Rev. D 47, 2504 (1993) arXiv:hep-th/9207043.

[24] E. Witten, "Mirror manifolds and topological field theory," arXiv:hep-th/9112056.

[25] R. Dijkgraaf, S. Gukov, A. Neitzke and C. Vafa, "Topological M-theory as unification of form theories of gravity," arXiv:hep-th/0411073.

[26] M. Bershadsky, S. Cecotti, H. Ooguri and C. Vafa, "Kodaira-Spencer theory of gravity and exact results for quantum string amplitudes," Commun. Math. Phys. 165, 311 (1994) arXiv:hep-th/9309140. 
[27] V. P. Nair, "A Current Algebra For Some Gauge Theory Amplitudes," Phys. Lett. B 214, 215 (1988).

[28] S. J. Parke and T. R. Taylor, "An Amplitude For N Gluon Scattering," Phys. Rev. Lett. 56, 2459 (1986); F. A. Berends and W. T. Giele, "Recursive Calculations For Processes With N Gluons," Nucl. Phys. B 306, 759 (1988); M. L. Mangano and S. J. Parke, "Multiparton Amplitudes In Gauge Theories," Phys. Rept. 200, 301 (1991).

[29] J. Park and S. J. Rey, "Supertwistor Orbifolds: Gauge Theory Amplitudes and Topological Strings," arXiv:hep-th/0411123. 